\title{
Blind Joint Maximum Likelihood Channel Estimation and Data Detection for SIMO Systems
}

\author{
Sheng Chen* Xiao-Chen Yang Lei Chen Lajos Hanzo \\ School of Electronics and Computer Science, University of Southampton, Southampton SO17 1BJ, UK
}

\begin{abstract}
A blind adaptive scheme is proposed for joint maximum likelihood (ML) channel estimation and data detection of singleinput multiple-output (SIMO) systems. The joint ML optimisation over channel and data is decomposed into an iterative optimisation loop. An efficient global optimisation algorithm called the repeated weighted boosting search is employed at the upper level to optimally identify the unknown SIMO channel model, and the Viterbi algorithm is used at the lower level to produce the maximum likelihood sequence estimation of the unknown data sequence. A simulation example is used to demonstrate the effectiveness of this joint ML optimisation scheme for blind adaptive SIMO systems.
\end{abstract}

Keywords: Blind space-time equalisation, single-input multiple-output (SIMO) systems, maximum likelihood (ML) estimation.

\section{Introduction}

The single-input multiple-output (SIMO) system, consisting of a single-antenna transmitter and a receiver equipped with multiple antennas, has enjoyed popularity owing to its simplicity. A space-time equaliser (STE) based on this SIMO structure is capable of mitigating the channel impairment arising from hostile multipath propagation. For the sake of improving the achievable system throughput, blind adaptation of the STE is attractive, since this avoids the reduction of the effective throughput by invoking training. Blind space-time equalisation of the SIMO system can be performed by directly adjusting the STE's parameters using the constant modulus algorithm (CMA) type adaptive scheme ${ }^{[1-5]}$. Blind space-time equalisation performance can further be improved by aiding the CMA with a soft decision-directed scheme ${ }^{[6]}$. Research for blind adaptive SIMO systems has also been focused on blind channel identification ${ }^{[7-9]}$. Once the SIMO channel impulse responses (CIRs) have been identified, various designs, such as the minimum mean square error or minimum bit error rate ${ }^{[10]}$, can be invoked for the STE. Alternatively, the decoupled weighted iterative least squares with projection (DW-ILSP) algorithm ${ }^{[11,12]}$ can be adopted. The DW-ILSP algorithm is a batch suboptimal EM-type algorithm, which iteratively performs channel estimation and symbol detection.

This contribution develops a blind adaptive scheme of joint maximum likelihood (ML) channel estimation and data detection for the SIMO system. The proposed algorithm decomposes the joint optimisation over channel and data into an iterative optimisation loop by combining a global optimisation method, referred to as the repeated weighted boosting search (RWBS $)^{[13]}$, for an optimal estimation of the SIMO channel and the Viterbi algorithm $(\mathrm{VA})^{[14]}$ for the maximum likelihood sequence estimation of the transmitted data sequence. Specifically, at the upper level, the RWBS algorithm searches the channel parameter space to optimise the ML criterion, while at the lower level,

Manuscript received date; revised date

*Corresponding author. E-mail address: sqc@ecs.soton.ac.uk the VA decodes data based on the given channel model and feeds back the corresponding likelihood metric to the RWBS algorithm. The effectiveness of this joint ML estimation scheme for blind equalisation of the SIMO system is demonstrated by a simulation example. We point out that a genetic algorithm (GA) can be used in place of the RWBS algorithm to optimise the SIMO channel estimate. In this case the proposed scheme becomes an extension of the joint ML channel and data estimation scheme using the GA originally developed for the single-input single-output (SISO) system $^{[15]}$.

\section{The proposed blind joint ML estima- tion algorithm}

Consider the SIMO system employing a single transmitter antenna and $L(>1)$ receiver antennas. The symbol-rate sampled antennas' outputs $x_{l}(k), 1 \leq l \leq L$, are given by

$$
x_{l}(k)=\sum_{i=0}^{n_{c}-1} c_{i, l} s(k-i)+n_{l}(k)
$$

where $n_{l}(k)$ is the complex-valued Gaussian white noise associated with the $l$ th channel and $E\left[\left|n_{l}(k)\right|^{2}\right]=2 \sigma_{n}^{2},\{s(k)\}$ is the transmitted symbol sequence and is assumed to take values from the quadrature phase shift keying (QPSK) symbol set $\{ \pm 1 \pm j\}$, and $c_{i, l}$ are the CIR taps associated with the $l$ th receive antenna. For notational simplicity, we have assumed that each of the $L$ channels has the same length of $n_{c}$. Let

$$
\begin{aligned}
\boldsymbol{x} & =\left[x_{1}(1) x_{1}(2) \cdots x_{1}(N) x_{2}(1) \cdots x_{L}(N)\right]^{\mathrm{T}} \\
\boldsymbol{s} & =\left[s\left(-n_{c}+2\right) \cdots s(0) s(1) \cdots s(N)\right]^{\mathrm{T}} \\
\boldsymbol{c} & =\left[\begin{array}{lll}
c_{0,1} & c_{1,1} \cdots c_{n_{c}-1,1} & c_{0,2} \cdots c_{n_{c}-1, L}
\end{array}\right]^{\mathrm{T}}
\end{aligned}
$$

be the vector of $N \times L$ received signal samples, the corresponding transmitted data sequence and the vector of the SIMO CIRs, respectively. The probability density function of the received data vector $\boldsymbol{x}$ conditioned on the SIMO CIR 
$\boldsymbol{c}$ and the symbol vector $\boldsymbol{s}$ is

$$
\begin{gathered}
p(\boldsymbol{x} \mid \boldsymbol{c}, \boldsymbol{s})=\frac{1}{\left(2 \pi \sigma_{n}^{2}\right)^{N L}} \times \\
e^{-\frac{1}{2 \sigma_{n}^{2}} \sum_{k=1}^{N} \sum_{l=1}^{L}\left|x_{l}(k)-\sum_{i=0}^{n_{c}-1} c_{i, l} s(k-i)\right|^{2}} .
\end{gathered}
$$

The joint ML estimate of $\boldsymbol{c}$ and $\boldsymbol{s}$ is obtained by maximising $p(\boldsymbol{x} \mid \boldsymbol{c}, \boldsymbol{s})$ over $\boldsymbol{c}$ and $\boldsymbol{s}$ jointly. Equivalently, the joint ML estimate is the minimum of the cost function

$$
J_{\mathrm{ML}}(\hat{\boldsymbol{c}}, \hat{\boldsymbol{s}})=\frac{1}{N} \sum_{k=1}^{N} \sum_{l=1}^{L}\left|x_{l}(k)-\sum_{i=0}^{n_{c}-1} \hat{c}_{i, l} \hat{s}(k-i)\right|^{2}
$$

namely

$$
\left(\hat{\boldsymbol{c}}^{*}, \hat{\boldsymbol{s}}^{*}\right)=\arg \left[\min _{\hat{\boldsymbol{c}}, \hat{\boldsymbol{s}}} J_{\mathrm{ML}}(\hat{\boldsymbol{c}}, \hat{\boldsymbol{s}})\right] .
$$

The joint minimisation process (7) can also be solved using an iterative loop first over the data sequences $\hat{\boldsymbol{s}}$ and then over all the possible channels $\hat{\boldsymbol{c}}$ :

$$
\left(\hat{\boldsymbol{c}}^{*}, \hat{\boldsymbol{s}}^{*}\right)=\arg \left[\min _{\hat{\boldsymbol{c}}}\left(\min _{\hat{\boldsymbol{s}}} J_{\mathrm{ML}}(\hat{\boldsymbol{c}}, \hat{\boldsymbol{s}})\right)\right] .
$$

The inner or lower-level optimisation can readily be carried out using the standard VA. In order to guarantee a joint ML estimate, the search algorithm used in the outer or upperlevel optimisation should be capable of finding a globaloptimal or near optimal channel estimate efficiently. We employ the RWBS guided random search algorithm ${ }^{[13]}$ to perform the outer optimisation task. The detailed RWBS algorithm is given in Appendix. The proposed blind joint ML optimisation scheme can now be summarised.

Outer level optimisation. The RWBS algorithm searches the SIMO channel parameter space to find a global optimal estimate $\hat{\boldsymbol{c}}^{*}$ by minimising the mean square error (MSE)

$$
J_{\mathrm{MSE}}(\hat{\boldsymbol{c}})=J_{\mathrm{ML}}\left(\hat{\boldsymbol{c}}, \tilde{\boldsymbol{s}}^{*}\right)
$$

Inner level optimisation. Given the channel estimate $\hat{\boldsymbol{c}}$, the VA provides the ML decoded data sequence $\tilde{\boldsymbol{s}}^{*}$, and feeds back the corresponding value of the likelihood metric $J_{\mathrm{ML}}\left(\hat{\boldsymbol{c}}, \tilde{\boldsymbol{s}}^{*}\right)$ to the upper level.

Let $C_{\mathrm{VA}}$ be the complexity of the VA required to decode a data sequence of $N \times L$ samples, and denote $N_{\mathrm{VA}}$ the total number of VA calls required for the RWBS algorithm to converge. The complexity of the proposed scheme is obviously $N_{\mathrm{VA}} \times C_{\mathrm{VA}}$. The RWBS algorithm is a simple yet efficient global search algorithm. In several global optimisation applications investigated in [13], including the blind joint ML channel estimation and data detection for the SISO system, the RWBS algorithm achieved a similar convergence speed as the GA and was seen to be more accurate than the GA. The RWBS algorithm has additional advantages of requiring minimum programming effort and having fewer algorithmic parameters that require to set.

\section{Simulation example}

In the simulation, the number of receiver antennas was $L=4$, the transmitted data symbols were QPSK, and the SIMO CIRs, listed in Table 1, were simulated. The length of data samples was $N=50$. In practice, the value of the likelihood metric $J_{\mathrm{MSE}}(\hat{\boldsymbol{c}})$ is all that the upper level optimiser can see, and the convergence of the algorithm can only be observed through the MSE (9). In simulation, the performance of the algorithm can also be assessed by the mean tap error defined as

$$
\mathrm{MTE}=\|\boldsymbol{c}-a \cdot \hat{\boldsymbol{c}}\|^{2}
$$

where

$$
a= \begin{cases}+1, & \text { if } \hat{\mathbf{c}} \rightarrow+\mathbf{c} \\ -1, & \text { if } \hat{\mathbf{c}} \rightarrow-\mathbf{c} \\ -j, & \text { if } \hat{\mathbf{c}} \rightarrow+j \mathbf{c} \\ +j, & \text { if } \hat{\mathbf{c}} \rightarrow-j \mathbf{c}\end{cases}
$$

Note that since $\left(\hat{\boldsymbol{c}}^{*}, \hat{\boldsymbol{s}}^{*}\right),\left(-\hat{\boldsymbol{c}}^{*},-\hat{\boldsymbol{s}}^{*}\right),\left(-j \hat{\boldsymbol{c}}^{*},+j \hat{\boldsymbol{s}}^{*}\right)$ and $\left(+j \hat{\boldsymbol{c}}^{*},-j \hat{\boldsymbol{s}}^{*}\right)$ are all the solutions of the joint ML estimation problem (7), the channel estimate $\hat{\boldsymbol{c}}$ can converges to $c,-c, j c$ or $-j c$.

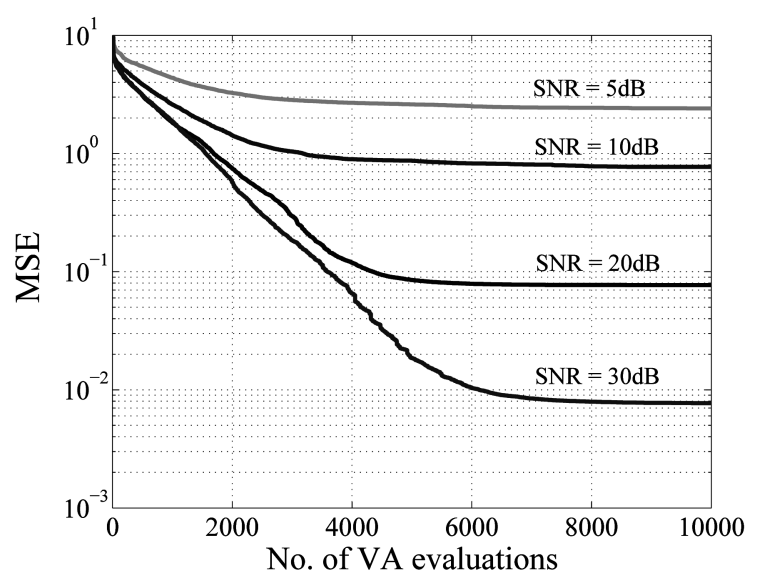

Fig. 1 MSE against number of VA evaluations averaged over 50 runs using the RWBS for the SIMO channel listed in Table 1. The length of data samples $N=50$.

Figs. 1 and 2 show the evolutions of the MSE and MTE averaged over 50 runs and for different signal to noise ratios (SNR), respectively, obtained by the proposed blind joint ML optimisation scheme using the RWBS. From Fig. 1, it can be seen that the MSE converges to the noise floor. Phase ambiguity of $90^{\circ}, 180^{\circ}$ or $270^{\circ}$ associated with the blind ML estimate for $\boldsymbol{s}$ cannot be resolved by the blind adaptive scheme itself. In practice, this ambiguity is resolved either by adopting differential encoding or by employing a few pilot training symbols. We adopted the complete blind adaptive scheme of using differential encoding. Fig. 3 depicts the bit error rate (BER) of the blind joint ML optimisation scheme with differential encoding, in comparison with the BERs of the optimal maximum likelihood sequence estimation in the known channel case with and without differential encoding. It is seen that the proposed blind scheme only induces half $\mathrm{dB}$ degradation in SNR compared with the optimal solution with differential encoding.

We also used the GA to perform the upper-level optimisation, and the results obtained by this GA-based blind 
Table 1 The simulated SIMO system

\begin{tabular}{cccr}
\hline Channel & \multicolumn{3}{c}{ Channel impulse response } \\
\hline 1 & $0.3652-0.2739 \mathrm{j}$ & $0.7304+0.1825 \mathrm{j}$ & $-0.4402+0.1761 \mathrm{j}$ \\
2 & $0.2783+0.2376 \mathrm{j}$ & $-0.6362+0.1039 \mathrm{j}$ & $0.6671-0.0741 \mathrm{j}$ \\
3 & $-0.6393+0.2494 \mathrm{j}$ & $-0.5169-0.3084 \mathrm{j}$ & $0.3651+0.1826 \mathrm{j}$ \\
4 & $-0.1539+0.6928 \mathrm{j}$ & $-0.5389-0.0770 \mathrm{j}$ & $0.2683-0.3578 \mathrm{j}$ \\
\hline
\end{tabular}



Fig. 2 MTE against number of VA evaluations averaged over 50 runs using the RWBS for the SIMO channel listed in Table 1. The length of data samples $N=50$.

joint ML estimation scheme are presented in Figs. 4 and 5. Comparing Fig. 1 with Fig. 4, it can be seen that both the RWBS and GA based schemes have similar convergence speed in terms of the total number of required VA evaluations. It can also be seen that the true estimation accuracy of the RWBS-based scheme is more accurate than that of the GA-based one, as confirmed by comparing Fig. 2 with Fig. 5.

All the above results were obtained under the assumption that the correct SIMO CIR length $n_{c}=3$ was known. In reality, the CIR length $n_{c}$ is unknown and has to be estimated. Fig. 6 shows the MSE of the blind joint ML scheme using the RWBS optimiser, as a function of the estimated channel length. It can be seen that the true channel length $n_{c}=3$ was correctly identified by the blind joint ML optimisation scheme.

\section{Conclusions}

A batch scheme using the global optimisation method, called the RWBS, has been developed for blind space-time equalisation of the SIMO system based on the joint ML channel estimation and data detection. The proposed algorithm provides the best performance over other types of blind adaptive schemes for SIMO systems at the expense of computational complexity. Our simulation study has shown that this blind joint ML optimisation scheme requires very few received data samples to achieve a near optimal solution of the maximum likelihood sequence estimation for data detection.



Fig. 3 Comparison of bit error rate performance using the ML sequence detection for the SIMO channel listed in Table 1. The length of data samples for the blind scheme is $N=50$.

\section{Appendix. Repeated weighted boosting search}

Solve the generic optimisation problem

$$
\min _{\boldsymbol{u} \in \mathcal{U}} J(\boldsymbol{u})
$$

where $\mathcal{U}$ defines the feasible set of $\boldsymbol{u}$, with the RWBS algorithm ${ }^{[13]}$. The algorithm is detailed in the following.

Specify the algorithmic parameters: population size $P_{S}$, number of generations in the repeated search $N_{G}$, and number of iterations in the weighted boosting search $N_{B}$.

Outer loop: generations For $\left(g=1 ; g \leq N_{G} ; g=g+1\right)$ \{

Generation initialisation: Initialise the population by setting $\boldsymbol{u}_{1}^{(g)}=\boldsymbol{u}_{\text {best }}^{(g-1)}$ and randomly generating rest of the population members $\boldsymbol{u}_{i}^{(g)}, 2 \leq i \leq P_{S}$, where $\boldsymbol{u}_{\text {best }}^{(g-1)}$ denotes the solution found in the previous generation. If $g=1, \boldsymbol{u}_{1}^{(g)}$ is also randomly chosen

Weighted boosting search initialisation: Assign the initial distribution weightings $\delta_{i}(0)=\frac{1}{P_{S}}, 1 \leq i \leq P_{S}$, for the population, and calculate the cost function value of each point

$$
J_{i}=J\left(\boldsymbol{u}_{i}^{(g)}\right), 1 \leq i \leq P_{S}
$$

Inner loop: weighted boosting search For $(t=1$; $\left.t \leq N_{B} ; t=t+1\right)\{$

Step 1. Boosting

1) Find

$$
i_{\text {best }}=\arg \min _{1 \leq i \leq P_{S}} J_{i} \text { and } i_{\text {worst }}=\arg \max _{1 \leq i \leq P_{S}} J_{i}
$$






Fig. 4 MSE against number of VA evaluations averaged over 50 runs using the GA for the SIMO channel listed in Table 1. The length of data samples $N=50$.

Denote $\boldsymbol{u}_{\text {best }}^{(g)}=\boldsymbol{u}_{i_{\text {best }}^{(g)}}$ and $\boldsymbol{u}_{\text {worst }}^{(g)}=\boldsymbol{u}_{i_{\text {worst }}^{(g)}}$

2) Normalise the cost function values

$$
\bar{J}_{i}=\frac{J_{i}}{\sum_{m=1}^{P_{S}} J_{m}}, 1 \leq i \leq P_{S}
$$

3) Compute a weighting factor $\beta_{t}$ according to

$$
\eta_{t}=\sum_{i=1}^{P_{S}} \delta_{i}(t-1) \bar{J}_{i}, \beta_{t}=\frac{\eta_{t}}{1-\eta_{t}}
$$

4) Update the distribution weightings for $1 \leq i \leq P_{S}$

$$
\delta_{i}(t)= \begin{cases}\delta_{i}(t-1) \beta_{t}^{\bar{J}_{i}}, & \text { for } \beta_{t} \leq 1 \\ \delta_{i}(t-1) \beta_{t}^{1-\bar{J}_{i}}, & \text { for } \beta_{t}>1\end{cases}
$$

and normalise them

$$
\delta_{i}(t)=\frac{\delta_{i}(t)}{\sum_{m=1}^{P_{S}} \delta_{m}(t)}, 1 \leq i \leq P_{S}
$$

Step 2. Parameter updating

1) Construct the $\left(P_{S}+1\right)$ th point using the formula

$$
\boldsymbol{u}_{P_{S}+1}=\sum_{i=1}^{P_{S}} \delta_{i}(t) \boldsymbol{u}_{i}^{(g)}
$$

2) Construct the $\left(P_{S}+2\right)$ th point using the formula

$$
\boldsymbol{u}_{P_{S}+2}=\boldsymbol{u}_{\text {best }}^{(g)}+\left(\boldsymbol{u}_{\text {best }}^{(g)}-\boldsymbol{u}_{P_{S}+1}\right)
$$

3) Compute the cost function values $J\left(\boldsymbol{u}_{P_{S}+1}\right)$ and $J\left(\boldsymbol{u}_{P_{S}+2}\right)$ for these two points and find

$$
i_{*}=\arg \min _{i=P_{S}+1, P_{S}+2} J\left(\boldsymbol{u}_{i}\right)
$$

4) The pair $\left(\boldsymbol{u}_{i_{*}}, J\left(\boldsymbol{u}_{i_{*}}\right)\right)$ then replaces $\left(\boldsymbol{u}_{\text {worst }}^{(g)}, J_{i_{\text {worst }}}\right)$ in the population



Fig. 5 MTE against number of VA evaluations averaged over 50 runs using the GA for the SIMO channel listed in Table 1. The length of data samples $N=50$.



Fig. 6 MSE as a function of the estimated SIMO channel length using the RWBS for the SIMO channel listed in Table 1. The length of data samples $N=50$.

\} End of inner loop The solution found in the $g$ th generation is $\boldsymbol{u}=\boldsymbol{u}_{\text {best }}^{(g)}$

F End of outer loop This yields the solution $\boldsymbol{u}=\boldsymbol{u}_{\mathrm{best}}^{\left(N_{G}\right)}$

The motivations and analysis of the RWBS algorithm as a global optimiser were detailed in [13]. To guarantee a global optimal solution as well as to achieve a fast convergence, the algorithmic parameters, $P_{S}, N_{G}$ and $N_{B}$, need to be set carefully. The appropriate values for these algorithmic parameters depend on the dimension of $\boldsymbol{u}$ and how hard the objective function is optimised. Generally, these algorithmic parameters have to be found empirically, just as in any global optimisation algorithm. The elitist initialisation is very useful, as it keeps the information obtained by the previous search generation, which otherwise would be lost due to the randomly sampling initialisation. In the inner loop optimisation, there is no need for every member of the population to converge to a (local) minimum, and it 
is sufficient to locate where the minimum lies. Thus, the number of weighted boosting iterations, $N_{B}$, can be set to a relatively small integer. This makes the search efficient, achieving convergence with a small number of the cost function evaluations. The population size $P_{S}$ and the number of generations $N_{G}$ should be chosen sufficiently large so that the parameter space is sampled sufficiently to guarantee a global-optimal solution.

\section{References}

[1] J. J. Shynk, R. P. Gooch. The Constant Modulus Array for Cochannel Signal Copy and Direction Finding. IEEE Transactions on Signal Processing, vol. 44, no. 3, pp. 652-660, 1996.

[2] J. J. Shynk, A. V. Keerthi, A. Mathur. Steady-state Analysis of the Multistage Constant Modulus Array. IEEE Transactions on Signal Processing, vol. 44, no. 4, pp. 948-962, 1996.

[3] H. H. Zeng, L. Tong, C. R. Johnson, Jr. Relationships between the Constant Modulus and Wiener Receivers. IEEE Transactions on Information Theory, vol. 44, no. 4, pp. 1523-1538, 1998.

[4] C. -Y. Chi, C. -Y. Chen, C. -H. Chen, C. -C. Feng. Batch Processing Algorithms for Blind Equalization Using Higher-order Statistics. IEEE Signal Processing Letters, vol. 20, no. 1, pp. 25-49, 2003

[5] K. Yang, T. Ohira, Y. Zhang, C. -Y. Chi. Super-exponential blind Adaptive Beamforming. IEEE Transactions on Signal Processing, vol. 52, no. 6, pp. 1549-1563, 2004.

[6] S. Chen, A. Wolfgang, L. Hanzo. Constant Modulus Algorithm Aided Soft Decision-directed Blind Space-time Equalization for SIMO Channels. In Proceedings of Vehicular Technology-2004 Fall, Los Angeles, USA, pp. 1718-1722, Sept. 26-29, 2004.

[7] H. Gazzah, P. A. Regalia, J. -P. Delmas. Asymptotic Eigenvalue Distribution of Block Toeplitz Matrices and Application to Blind SIMO Channel Identification. IEEE Transactions on Information Theory, vol. 47, no. 3, pp. 1243-1251, 2001.

[8] D. Luengo, I. Santamaria, J. Ibanez, L. Vielva, C. Pantaleon. A Fast Blind SIMO Channel Identification Algorithm for Sparse Sources. IEEE Signal Processing Letters, vol. 10, no. 5, pp. 148-151, 2003.

[9] I. Santamaria, J. Via, C. C. Gaudes. Robust Blind Identification of SIMO Channels: A Support Vector Regression Approach. In Proceedings of IEEE International Conference on Acoustics Speech, and Signal Processing, Montreal, Canada, vol. 5, pp. 673-676, May 17-21, 2004.

[10] A. Livingstone, S. Chen. Adaptive Space-time Equalisation for Multiple-antenna Assisted Multiple-input Multiple-output Systems. In Proceedings of 2nd IEE/EURASIP Conference on DSPenabledRadio, Southampton, UK, pp. 9/1-9/7, Sept. 1920, 2005.

[11] A. Ranheim. A Decoupled Approach to Adaptive Signal Separation Using an Antenna Array. IEEE Transactions on Vehicular Technology, vol. 48, no. 3, pp. 676-682, 1999.

[12] A. Dogandzic, A. Nehorai. Generalized Multivariate Analysis of Variance - A Unified Framework for Signal Processing in Correlated Noise. IEEE Signal Processing Magazine, vol. 20, no. 5, pp. 39-54, 2003.

[13] S. Chen, X. X. Wang, C. J. Harris. Experiments with Repeating Weighted Boosting Search for Optimization in Signal Processing Applications. IEEE Transactions on Systems, Man, and Cybernetics, Part B, vol. 35, no. 4, pp. 682-693, 2005.

[14] J. G. Proakis. Digital Communications. 3rd ed., McGraw-Hill, New York, 1995.

[15] S. Chen, Y. Wu. Maximum Likelihood Joint Channel and Data Estimation Using Genetic Algorithms. IEEE Transactions on Signal Processing, vol. 46, no. 5, pp. 1469-1473, 1998.



Sheng Chen received his B. Eng degree in control engineering from the East China Petroleum Institute, Dongying, China, in 1982 , and the Ph. D. degree in control engineering from the City University, London, in 1986. In 2005, he was awarded the Doctor of Sciences (DSc) degree by the University Southampton, UK. Since 1999 he has been with the School of Electronics and Computer Science, the University of Southampton, UK. He previously held research and academic appointments at the Universities of Sheffield, Edinburgh and Portsmouth, all in UK.

He has published over 260 research papers. His recent research interests include adaptive signal processing, wireless communications, modelling and identification of nonlinear systems, neural network and machine learning, finite-precision digital controller design, evolutionary computation methods, and optimization.

Dr. Chen is a senior member of IEEE. In the database of the world's most highly cited researchers in various disciplines, compiled by Institute for Scientific Information (ISI) of the USA, Dr Chen is on the list of the highly cited researchers in the engineering category.

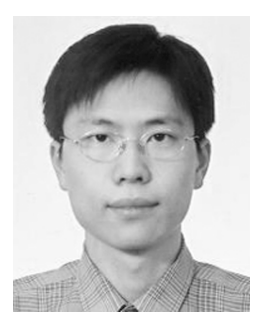

Xiao-Chen Yang received his B. Sc. degree in electrical engineering from Tsinghua University, China, in 2003, and the M. Sc. degree in wireless communications from the University of Southampton, UK, in 2004. He is currently studying in Civil Engineering Department at the University of Washington, Seattle, USA.



Lei Chen received his B.Sc. degree from Zhejiang University, China, in 2004, and the M.Sc. degree in wireless communications from the University of Southampton, UK, in 2005. Since 2006, he has been pursuing the Ph. D. degree at Institute of Digital Communications, School of Engineering and Electronics, the University of Edinburgh, UK.

His current research interests include signal processing and wireless communications, with emphasis on identification of time-varying system, channel estimation and equalisation, and their applications in wireless communications.

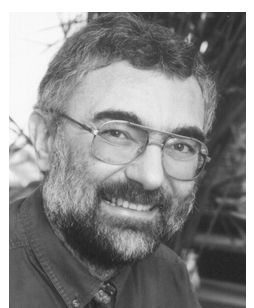

Lajos Hanzo received his M.Sc. degree in Lajos Hanzo received his M.Sc. degree in
electronics in 1976 and the Ph. D. degree in 1983. In 2004 he was awarded the Doctor of Sciences (DSc) degree by the University Southampton, UK. During his 28-year career in telecommunications he has held various research and academic posts in Hungary, Germany and UK. Since 1986 he has been with the School of Electronics and Computer Science, the University of Southampton, UK, where he holds the chair in telecommunications.

He has co-authored 11 John Wiley/IEEE Press books totalling about 9000 pages on mobile radio communications, published in excess of 550 research papers, organised and chaired conference sessions, presented overview lectures and has been awarded a number of distinctions. He is an enthusiastic supporter of industrial-academic liaison. He also offers a range of industrial research overview courses.

Prof. Hanzo is a Fellow of the Royal Academy of Engineering (FREng), UK. He is an IEEE Distinguished Lecturer of both the Communications Society and the Vehicular Technology Society as well as a Fellow of both the IEEE and IEE. He is a non-executive director of the Virtual Centre of Excellence (VCE) in mobile communications, UK, a governor of the IEEE VT society and an executive board member of the Pan-European Network of Excellence known as NEWCOM. 